\title{
Largest Placement of One Convex Polygon Inside Another*
}

\author{
P. K. Agarwal, ${ }^{1}$ N. Amenta, ${ }^{2 \dagger}$ and M. Sharir ${ }^{3}$ \\ ${ }^{1}$ Department of Computer Science, Box 90129, Duke University, \\ Durham, NC 27708-0129, USA \\ pankaj@euclid.cs.duke.edu \\ ${ }^{2}$ Xerox PARC, 3333 Coyote Hill Road, \\ Palo Alto, CA 94304, USA \\ ${ }^{3}$ School of Mathematical Sciences, Tel Aviv University, \\ Tel Aviv 69978, Israel \\ sharir@math.tau.ac.il \\ and \\ Courant Institute of Mathematical Sciences, New York University, \\ New York, NY 10012, USA
}

\begin{abstract}
We show that the largest similar copy of a convex polygon $P$ with $m$ edges inside a convex polygon $Q$ with $n$ edges can be computed in $O\left(m n^{2} \log n\right)$ time. We also show that the combinatorial complexity of the space of all similar copies of $P$ inside $Q$ is $O\left(m n^{2}\right)$, and that it can also be computed in $O\left(m n^{2} \log n\right)$ time.
\end{abstract}

Let $P$ be a convex polygon with $m$ edges and let $Q$ be a convex polygon with $n$ edges. Our goal is to find the largest similar copy of $P$ inside $Q$ (allowing translation, rotation, and scaling of $P$ ); see Fig. 1. A restricted version of this problem, in which we just determine whether $P$ can be placed inside $Q$ without scaling, was solved by Chazelle [6], in $O\left(m n^{2}\right)$ time. See also [1], [8], and [16] for other approaches to the more general

\footnotetext{
* Pankaj Agarwal and Micha Sharir have been supported by a grant from the U.S.-Israeli Binational Science Foundation. Pankaj Agarwal has also been supported by National Science Foundation Grant CCR93-01259, by Army Research Office MURI Grant DAAH04-96-1-0013, by a Sloan fellowship, and by an NYI award and matching funds from the Xerox Corporation. Nina Amenta was employed by the Geometry Center, which is officially the NSF Center for Computation and Visualization of Geometric Structures, supported by NSF/DMS-8920161. Micha Sharir has also been supported by NSF Grants CCR-91-22103 and CCR93-11127, by a Max-Planck Research Award, and by grants from the Israel Science Fund administered by the Israeli Academy of Sciences, and the G.I.F., the German-Israeli Foundation for Scientific Research and Development.

$\dagger$ Current address: Department of Computer Science, University of Texas-Austin, Austin, TX 78712, USA. amenta@cs.utexas.edu.
} 


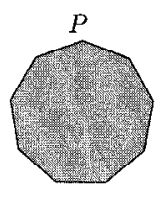

(i)

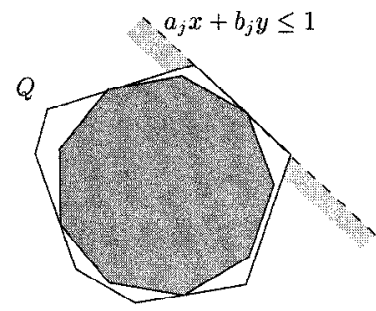

(ii)

Fig. 1. (i) The polygon $P$. (ii) The polygon $Q$ and a largest copy of $P$ inside $Q$.

problem, in which $Q$ is an arbitrary polygonal region. We remark that the complexity of the algorithms for the general case is considerably higher, about $O\left(m^{2} n^{2}\right)$ in [1], $O\left(m^{3} n^{2}\right)$ in [16], and $O\left(m^{4} n^{2}\right)$ in [8]. See [2], [3], [9], [12], and the references therein for other related work on the polygon-placement problem.

Problems concerning the placement of one polygon inside another are important in robotics and manufacturing. This restricted problem is also applicable to an approach to object recognition recently proposed by Basri and Jacobs [5], based on matching two-dimensional faces of polyhedral objects. The transformation that places the largest similar copy of a polygon $P$, derived from a face of an object model, inside a polygon $Q$, derived from an image, is a candidate for a transformation which matches the entire model to the image.

The geometric setup of the problem is as follows. We observe, following Baird [4], that similar placements of $P$ can be parametrized nicely by referring to an arbitrarily chosen reference point $p \in P$. A placement $\pi$ is represented by a quadruple $(s, t, u, v)$, where $(u, v)$ is a translation of $p$ in the plane, and $s=\rho \cos \theta, t=\rho \sin \theta$, where $P$ is rotated by $\theta$ and scaled by $\rho$, around $p$. Let $P_{\pi}$ denote the similar copy of $P$ corresponding to the placement $\pi$. The standard placement puts $p$ at the origin, with $\rho=1, \theta=u=v=0$. Thus if $(x, y)$ is a vertex of $P$ in the standard placement, its position at the placement $(s, t, u, v)$ is $(s x-t y+u, t x+s y+v)$. Such a placement of $P$ lies fully within $Q$ if and only if every vertex $\left(x_{i}, y_{i}\right)$ of $P$ lies in every half-space $a_{j} x+b_{j} y \leq 1$ containing $Q$ and bounded by the line supporting an edge of $Q$; see Fig. 1. That is, the placement $(s, t, u, v)$ must satisfy the following system of $m n$ linear inequalities:

$$
a_{j}\left(s x_{i}-t y_{i}+u\right)+b_{j}\left(t x_{i}+s y_{i}+v\right) \leq 1
$$

or

$$
L_{i, j}: \quad\left(a_{j} x_{i}+b_{j} y_{i}\right) s+\left(-a_{j} y_{i}+b_{j} x_{i}\right) t+a_{j} u+b_{j} v \leq 1 .
$$

In other words, the space $\mathcal{C}$ of all similar placements of $P$ inside $Q$ is a four-dimensional convex polyhedron formed by the intersection of $m n$ half-spaces. This already implies that the combinatorial complexity of $\mathcal{C}$ is $O\left(m^{2} n^{2}\right)$, and that it can be constructed in $O\left(m^{2} n^{2}\right)$ time [15]. However, we improve this bound in what follows, exploiting the fact that $\mathcal{C}$ is highly degenerate.

In order to find the largest similar copy of $P$ inside $Q$, we need to find a point of $\mathcal{C}$ that maximizes $s^{2}+t^{2}=\rho^{2}$. Unfortunately, maximizing a convex function over a convex 
polyhedral domain is not an LP-type problem (in the setup of [11], where a linear-time randomized solution for such problems is described), so it appears that the algorithm of choice is to examine each vertex of $\mathcal{C}$ and select the one with the largest value of $s^{2}+t^{2}$ (the maximum of such a convex function is clearly attained at a vertex of $\mathcal{C}$ ). Moreover, since $s^{2}+t^{2}$ depends only on $s$ and $t$, it suffices to project $\mathcal{C}$ onto the $s t$-plane, and examine only the vertices of that projection.

The main result of the paper is the following theorem.

\section{Theorem 1.}

(a) The total number of vertices of $\mathcal{C}$ is $O\left(m n^{2}\right)$, and they can all be computed in time $O\left(m n^{2} \log n\right)$.

(b) The vertices of the projection of $\mathcal{C}$ onto the st-plane can be computed in time $O\left(m n^{2} \log n\right)$.

Remark. Although part (b) follows immediately from part (a), we give a direct proof of (b), which is somewhat simpler and provides more geometric insight into the structure of the problem.

Proof of Theorem 1. We prove both parts by applying the standard duality transform that maps a point $\left(\xi_{1}, \xi_{2}, \xi_{3}, \xi_{4}\right)$ to the hyperplane $\xi_{1} s+\xi_{2} t+\xi_{3} u+\xi_{4} v=1$ and vice versa. We denote the coordinates in the dual space by $s^{*}, t^{*}, u^{*}, v^{*}$. For $1 \leq i \leq m$ and $1 \leq j \leq n$, let $w_{i, j}$ denote the point dual to the hyperplane bounding the half-space $L_{i, j}$, i.e.,

$$
w_{i, j}=\left(a_{j} x_{i}+b_{j} y_{i},-a_{j} y_{i}+b_{j} x_{i}, a_{j}, b_{j}\right) .
$$

The convex hull of the points in $\left\{w_{i, j} \mid 1 \leq i \leq m, 1 \leq j \leq n\right\}$, denoted by $\mathcal{D}$, is the dual polytope of $\mathcal{C}$. It is easy to verify that all the points $w_{i, j}$ are extreme points of $\mathcal{D}$ (or, equivalently, that all the hyperplanes bounding the half-spaces $L_{i, j}$ contain facets of $\mathcal{C}$ ). Note that, for each fixed $j$ corresponding to an edge of $Q$, the convex hull $G_{j}$ of $\left\{w_{i, j}\right\}_{i=1}^{m}$ is a similar copy of $P$ that lies in the 2-plane $\pi_{j}: u^{*}=a_{j}, v^{*}=b_{j}$. The dual polytope $\mathcal{D}$, then, is the convex hull of $n$ similar copies of $P$, placed in parallel 2-planes in 4-space. Each facet of $\mathcal{D}$ corresponds to a placement $\pi$ of $P$ inside $Q$ such that $P_{\pi} \subseteq Q$ and there are at least four vertex-edge incidences between the vertices of $P_{\pi}$ and the edges of $Q$.

We begin with the proof of part (b). We exploit the well-known fact that projection in the primal is slicing in the dual. In more detail, let $\mathcal{C}_{2}$ denote the projection of $\mathcal{C}$ onto the $s t$-plane $u=0, v=0$, as effected by the mapping $(s, t, u, v) \mapsto(s, t, 0,0)$. Then a line $\alpha s+\beta t=1$ in the $s t$-plane is a supporting line of $\mathcal{C}_{2}$ if and only if the hyperplane $\alpha s+\beta t=1$ is a supporting hyperplane of $\mathcal{C}$ in $\mathbb{R}^{4}$. This is equivalent, in the dual, to having the point $(\alpha, \beta, 0,0)$ belong to the boundary of $\mathcal{D}$. Thus, computing $\mathcal{C}_{2}$ is equivalent to computing the cross section $\mathcal{D}_{2}$ of $\mathcal{D}$ with the 2-plane $u^{*}=0, v^{*}=0$.

Our strategy for computing $\mathcal{D}_{2}$ is first to compute $\mathcal{D}_{3}$, the cross section of $\mathcal{D}$ with the hyperplane $u^{*}=0$, and then to slice $\mathcal{D}_{3}$ with the plane $v^{*}=0$. Since it is trivial to intersect a three-dimensional polytope with a plane, in time proportional to the complexity of the polytope, we only consider the construction of $\mathcal{D}_{3}$. 


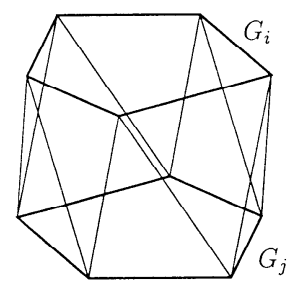

Fig. 2. Convex hull of parallel polygons.

Without loss of generality, we can assume that none of the $a_{j}$ 's is 0 . Then each of the polygons $G_{j}$ lies outside the hyperplane $u^{*}=0$. Hence, any vertex $w$ of $\mathcal{D}_{3}$ must be an intersection of $u^{*}=0$ with an edge of $\mathcal{D}$, connecting two vertices of a pair of distinct polygons, $G_{i}$ and $G_{j}$, where $G_{i}$ lies above $u^{*}=0$ and $G_{j}$ lies below. Moreover, $w$ must also be a vertex of the intersection of the convex hull of $G_{i} \cup G_{j}$ with $u^{*}=0$. So we can construct $\mathcal{D}_{3}$ by taking the convex hull, in $\mathbb{R}^{4}$, of every pair of polygons $G_{i}, G_{j}$, intersecting all of these subhulls with $u^{*}=0$, and then taking the convex hull of the resulting intersections.

We consider the geometry of the subhull of one such pair $G_{i}, G_{j}$. The two parallel 2-planes $u^{*}=a_{i}, v^{*}=b_{i}$ and $u^{*}=a_{j}, v^{*}=b_{j}$ lie in the common 3-plane $F_{i, j}$ defined by

$$
\left(b_{j}-b_{i}\right) u^{*}+\left(a_{i}-a_{j}\right) v^{*}+\left(b_{i} a_{j}-b_{j} a_{i}\right)=0
$$

and so does the subhull determined by $G_{i}, G_{j}$. The three-dimensional geometry of $\operatorname{conv}\left(G_{i} \cup G_{j}\right)$ in $F_{i, j}$ is as shown in Fig. 2 .

The intersection of $F_{i, j}$ with $u^{*}=0$ is the 2-plane

$$
u^{*}=0, \quad v^{*}=\frac{b_{i} a_{j}-b_{j} a_{i}}{a_{j}-a_{i}},
$$

which is also parallel to the two polygons $G_{i}, G_{j}$. Slicing the convex hull of the two parallel polygons with a parallel plane, we get a third parallel polygon $G_{i, j}$ which is the Minkowski sum of appropriately scaled copies of $G_{i}$ and $G_{j}$. This polygon has at most $2 m$ vertices, and it is easy to compute these vertices directly from the vertices of $G_{i}$ and $G_{j}$. Note that $G_{i, j}$ lies in both $F_{i, j}$ and in $u^{*}=0$.

The 3-polytope $\mathcal{D}_{3}$ in $u^{*}=0$ is the convex hull of all these polygons $G_{i, j}$. There are $O\left(n^{2}\right)$ such polygons, each with at most $2 m$ vertices, so the total complexity of $\mathcal{D}_{3}$ is $O\left(m n^{2}\right)$ (which of course is also a consequence of the bound for the overall complexity of $\mathcal{D}$, as asserted in part (a) and proven below).

The algorithm is simply to form the polygons $G_{i, j}$, take their three-dimensional convex hull, and intersect it with $v^{*}=0$. Since the Minkowski sum of two convex polygons can be computed in linear time [10], we spend $O\left(m n^{2}\right)$ time in computing the polygons $G_{i, j}$. Their convex hull can be computed in $O\left(m n^{2} \log n\right)$ time, using the divide-and-conquer algorithm of [14] (which now has only $O(\log n)$ recursive levels, because we start with the already available polygons $\left.G_{i, j}\right)$. Hence, the total running time is $O\left(m n^{2} \log n\right)$.

This completes the proof of part (b). Note that in practical terms, the implementation 
of this algorithm is a straightforward setup followed by a three-dimensional convex hull computation, which can be performed efficiently with publicly available software. ${ }^{1}$

We now return to the proof of part (a). We first consider the facets of $\mathcal{D}$ whose supporting hyperplanes are parallel to the 2-plane $u^{*}=0, v^{*}=0$. The equation of such a hyperplane $h_{F}$ of a facet $F$ has the form $\beta u^{*}+\gamma v^{*}+\delta=0$. Hence, if $h_{F}$ contains a vertex of some $G_{j}$, it must contain the entire polygon $G_{j}$. It then follows that $F$ must be the convex hull of the union of two polygons $G_{i}, G_{j}$ (as in the proof of part (b) given above). The facet $F$ is dual to the placement of $P$ in which it is shrunk to a point and all its vertices are incident to the vertex of $Q$ where edge $i$ of $Q$ meets edge $j$ of $Q$ (so these two edges must be consecutive edges of $Q$ ). The number of such placements is $n$, and the complexity of each of the corresponding facets is $O(m)$, since it is the three-dimensional convex hull of $2 m$ points. (It is easily verified that each of these hulls is indeed a facet of $\mathcal{D}$.) It follows that the overall complexity of these facets of $\mathcal{D}$ is $O(m n)$. Constructing all these facets is easy to do in $O(m n)$ time.

Next, consider the facets of $\mathcal{D}$ whose supporting hyperplanes are not parallel to the 2plane $u^{*}=0, v^{*}=0$. Let $F$ be such a facet of $\mathcal{D}$, and let $h$ be the hyperplane supporting $F$. The equation of $h$ can be written as $t^{*}=\alpha s^{*}+\beta u^{*}+\gamma v^{*}+\delta$ (for simplicity we assume, without loss of generality, that $\alpha$ is never infinite). Then, for each $j=1, \ldots, n$, the line $\ell_{j}$ of intersection between $h$ and the 2-plane $\pi_{j}$ containing $G_{j}$ either touches or is disjoint from $G_{j}$. The equation of $\ell_{j}$ is $t^{*}=\alpha s^{*}+\beta a_{j}+\gamma b_{j}+\delta, u^{*}=a_{j}, v^{*}=b_{j}$. Note that the coefficient $\alpha$ uniquely determines the vertex of $G_{j}$ nearest to $\ell_{j}$, for every $j$, unless $\alpha$ is a "critical" value equal to the slope of an edge of some $G_{j}$. There are $v=m n$ such critical slopes $\alpha$, corresponding to the orientations at which an edge of $P$ is parallel to an edge of $Q$, and it is easy to compute them, in order, in time $O(m n \log n)$. Let $\alpha_{1}<\alpha_{2}<\cdots<\alpha_{\nu}$ be these critical slopes.

Let $K$ be an open interval of $\alpha$-coefficients between two successive critical slopes. Then, for each $j=1, \ldots, n$, there exists a unique vertex $w_{i(K), j}$ of $G_{j}$, such that if $h$ is any supporting hyperplane of $\mathcal{D}$ whose $\alpha$-coefficient lies in $K$, then $h$ can touch $G_{j}$, if at all, only at $w_{i(K), j}$. In other words, such an $h$ is also a supporting hyperplane of $S_{K}=\left\{w_{i(K), j}\right\}_{j=1}^{n}(h$ must of course touch at least one of these vertices, and at least four if it contains a facet of $\mathcal{D}$ ). For two adjacent intervals $K$ and $K^{\prime}$, the set $S_{K^{\prime}}$ is obtained from $S_{K}$ by replacing one vertex $w$ by another vertex $w^{\prime}$ (both being adjacent vertices of some $G_{j}$ ). It easily follows that every facet $F$ of $\mathcal{D}$ not parallel to $u^{*}=0, v^{*}=0$ is either a facet of $\operatorname{conv}\left(S_{K}\right)$, for some interval $K$, or, if the $\alpha$-coefficient of $F$ is a critical value, a facet of $\operatorname{conv}\left(S_{K} \cup S_{K^{\prime}}\right)$, for some pair of consecutive intervals $K$ and $K^{\prime}$. If the vertices of $P$ and $Q$ are in general position, these latter facets correspond to placements in which an edge of $P$ is incident to an edge of $Q$. In fact, we can prove the following stronger claim. Assuming $\alpha_{0}=-\infty$ and $\alpha_{v+1}=+\infty$, let $K_{i}$ be the open interval $\left(\alpha_{i}, \alpha_{i+1}\right)$, for $0 \leq i \leq v$. With a slight abuse of notation, let $S_{i}=S_{K_{i}}$ and let $\xi_{i}$ denote the unique element of $S_{i} \backslash S_{i-1}$, for $1 \leq i \leq v$.

\footnotetext{
${ }^{1}$ For example, Ken Clarkson's hull program, at http://netlib.att.com/netlib/voronoi/ hull.html, or Ioannis Emiris' chD, available by ftp from robotics.eecs.Berkeley.edu in /pub/ConvexHull. These and other convex hull programs are listed on the computational geometry software Web page at http://www.geom. umn. edu/software/cglist. Using either of these programs gives a randomized algorithm which runs in time $O\left(m n^{2} \log m n\right)$, slightly worse than our theoretical result.
} 
Lemma 2. Every facet $F$ of $\mathcal{D}$ that is not parallel to $u^{*}=0, v^{*}=0$ is either a facet of the convex hull conv $\left(S_{0}\right)$ or a facet of the convex hull conv $\left(S_{i-1} \cup\left\{\xi_{i}\right\}\right)$ incident to $\xi_{i}$ for some $1 \leq i \leq v$.

Proof. Let $F$ be a facet of $\mathcal{D}$ that is not parallel to $u^{*}=0, v^{*}=0$ and that is not a facet of $\operatorname{conv}\left(S_{0}\right)$. Let $W$ be the set of vertices of $F$, and let $i \leq v$ be the index such that the $\alpha$-coefficient of the hyperplane supporting $F$ lies in the (semi-open) interval $\left(\alpha_{i-1}, \alpha_{i}\right]$. Then, by the above argument, $W \subseteq S_{i-1} \cup\left\{\xi_{i}\right\}$. Suppose $j \leq i$ is the largest index such that $\xi_{j} \in W$ (i.e., $S_{j}$ is obtained from $S_{j-1}$ by inserting one of the points of $W$ and deleting a point of $S_{j-1}$ ). Then it is easily seen that $W \subseteq S_{j-1} \cup\left\{\xi_{j}\right\}$. Hence, $F$ is a facet of $\operatorname{conv}\left(S_{j-1} \cup\left\{\xi_{j}\right\}\right)$ incident to $\xi_{j}$, as asserted.

This lemma suggests that we should compute $\operatorname{conv}\left(S_{0}\right)$ and, for each $1 \leq i \leq v$, we compute the facets of $\operatorname{conv}\left(S_{i-1} \cup\left\{\xi_{i}\right\}\right)$ incident to $\xi_{i}$. Since the hyperplanes containing the facets of $\operatorname{conv}\left(S_{i-1} \cup\left\{\xi_{i}\right\}\right)$ incident to $\xi_{i}$ have only three degrees of freedom, this problem can be formulated as a three-dimensional convex hull problem, and can be solved in $O(n \log n)$ time; the number of these facets, as well as their overall complexity, is $O(n)$. Notice that the set $S_{0}$ and the vertices $\xi_{i}$, for $1 \leq i \leq v$, can be computed in $O(m n \log n)$ time. Repeating this algorithm for all $1 \leq i \leq v$ and computing $\operatorname{conv}\left(S_{0}\right)$, the algorithm produces a total of $O\left(m n^{2}\right)$ facets, of $O\left(m n^{2}\right)$ overall complexity, in time $O\left(m n^{2} \log n\right)$.

These arguments prove that the total number of facets of $\mathcal{D}$ is $O\left(m n^{2}\right)$, and that their overall complexity, and hence the overall complexity of $\mathcal{C}$, is $O\left(m n^{2}\right)$. Unfortunately, the algorithm might produce additional spurious facets, which are not facets of $\mathcal{D}$. Indeed, a facet $F$ of $\operatorname{conv}\left(S_{i-1} \cup\left\{\xi_{i}\right\}\right)$ corresponds to a placement $\pi$ of $P$ such that there are at least four vertex-edge incidences between the vertices of $P_{\pi}$ and the edges of $Q$, and $F$ is spurious if $P_{\pi} \nsubseteq Q$. If the $\alpha$-coefficient of $F$ lies in the interval $K_{i-1} \cup K_{i}$, then it follows by definition that $F$ cannot be spurious. However, if this $\alpha$-coefficient lies in another interval $K_{j}$, for some $j \notin\{i-1, i\}$, then $F$ may be spurious because $P_{\pi}$ may violate a constraint $L_{u, v}$ corresponding to some vertex $w_{u, v} \in S_{j} \backslash\left(S_{i-1} \cup S_{i}\right)$. See Fig. 3 for an example: Let $\alpha_{i}$ be the critical slope at which the edge $p_{1} p_{2}$ of $P$ is parallel to the edge $e_{5}$ of $Q$. Then, by construction, $S_{i-1}=\left\{w_{6,1}, w_{5,2}, w_{4,3}, w_{3,4}, w_{1,5}, w_{7,6}\right\}$ and $\xi_{i}=w_{2,5}$. It is easy to verify that $\operatorname{conv}\left(\left\{w_{5,2}, w_{4,3}, w_{3,4}, w_{2,5}\right\}\right)$ is a facet of $S_{i-1} \cup\left\{\xi_{i}\right\}$ incident to $\xi_{i}=w_{2,5}$, but, as shown in Fig. 3, the corresponding copy of $P$ does not lie inside $Q$ (this facet is "violated" by $w_{7,1}$ ).

Hence, to complete our algorithm, we need to detect and discard the facets of the hulls $\operatorname{conv}\left(S_{K}\right)$ which are not facets of $\mathcal{D}$. This is accomplished as follows. We triangulate each computed facet $F$ into $O(|F|)$ tetrahedra, using the bottom-vertex triangulation scheme described in [7]. Let $\Delta$ denote the set of resulting tetrahedra; $|\Delta|=O\left(m n^{2}\right)$. Let $\mathcal{D}^{*}$ be the bottom-vertex triangulation of the boundary of $\mathcal{D}$. We want to discard those tetrahedra of $\Delta$ that are not facets of $\mathcal{D}^{*}$. For a vertex $w$, let $\Delta_{w} \subseteq \Delta$ be the subset of tetrahedra incident to $w$, and let $V_{w}$ be the set of vertices of the tetrahedra in $\Delta_{w}$. It is easily verified that a tetrahedron $\Delta \in \Delta_{w}$ is a facet of $\mathcal{D}^{*}$ if and only if $\Delta$ is a tetrahedron in the bottom-vertex triangulation of the boundary of $\operatorname{conv}\left(V_{w}\right)$, which is necessarily incident to $w$. We therefore compute the facets of $\operatorname{conv}\left(V_{w}\right)$ that are incident to $w$, by the reduction, noted above, to a three-dimensional convex hull construction, and then 


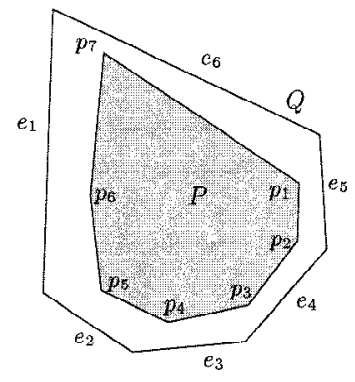

(i)

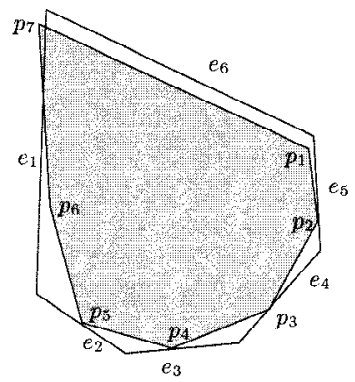

(ii)

Fig. 3. Spurious facets generated by the algorithm: (i) the orientation of $P$ lies in $K_{i-1}$, where $\xi_{i}=w_{2,5}$, and (ii) a placement of $P$ corresponding to a spurious facet of $S_{i-1} \cup\left\{w_{2,5}\right\}$.

compute the bottom-vertex triangulation of each such facet. Note that these facets can be computed in $O\left(\left|V_{w}\right| \log n\right)$ time, since the vertices of $V_{w}$ lie on only $n$ 2-planes, so that the convex hull computation requires only $O(\log n)$ recursive levels; we omit the easy details. We can now discard those tetrahedra in $\Delta_{w}$ that do not lie on the boundary of $\operatorname{conv}\left(V_{w}\right)$. Repeating this procedure for all vertices $w$ of $\mathcal{D}$ eliminates all spurious facets computed by the algorithm.

The running time of this step is $\sum_{w} O\left(\left|V_{w}\right| \log n\right)$, where the sum extends over all vertices $w$ of $\mathcal{D}$. Since $\sum_{w}\left|V_{w}\right|=4|\Delta|=O\left(m n^{2}\right)$, the total time spent is $O\left(m n^{2} \log n\right)$.

This completes the proof of part (a).

An immediate corollary of Theorem 1(b) is the following.

Corollary 3. The largest similar copy of $P$ inside $Q$ can be computed in $O\left(m n^{2} \log n\right)$ time.

We conclude this paper by constructing a pair of polygons $P$ and $Q$, with $m$ and $n$ vertices, respectively, so that there are $\Omega\left(m n^{2}\right)$ placements of $P$ inside $Q$, each of which induces four incidences of the form $(p, e)$, where $p$ is a vertex of $P$ and $e$ is an edge of $Q$. This implies that the combinatorial bound of Theorem 1(a) is tight in the worst case.

The construction is depicted in Fig. 4. Let $n$ be of the form $2 l+2$, for some positive integer $l, m$ an even integer, and $o$ the origin. The first $n / 2$ vertices $q_{1}, \ldots, q_{n / 2}$ of $Q$ are evenly distributed along the arc of the unit-radius circle, centered at $o$, which goes from $-\pi / 6$ to $\pi / 6$ (in counterclockwise direction). The vertices $q_{n / 2+1} \cdots q_{n}$ are evenly distributed along a tiny arc of a larger circle, say the circle with radius $10+\varepsilon$ and center $(10,0)$, and we let the tiny arc span the orientations between $\pi-\varepsilon / 2(10+\varepsilon)$ and $\pi+\varepsilon / 2(10+\varepsilon)$, so that its arc length is $\varepsilon$. The value of $\varepsilon$ will be chosen sufficiently small, in a manner to be detailed in a moment.

We place one vertex $p_{m}$ of $P$ at the origin $o$ and the remaining $m-1$ vertices, equally spaced, on a circular arc of radius $\frac{1}{4}$, centered at $\left(\frac{3}{4}, 0\right)$, that spans the orientations between $-\pi / 40 l$ and $+\pi / 40 l$. 


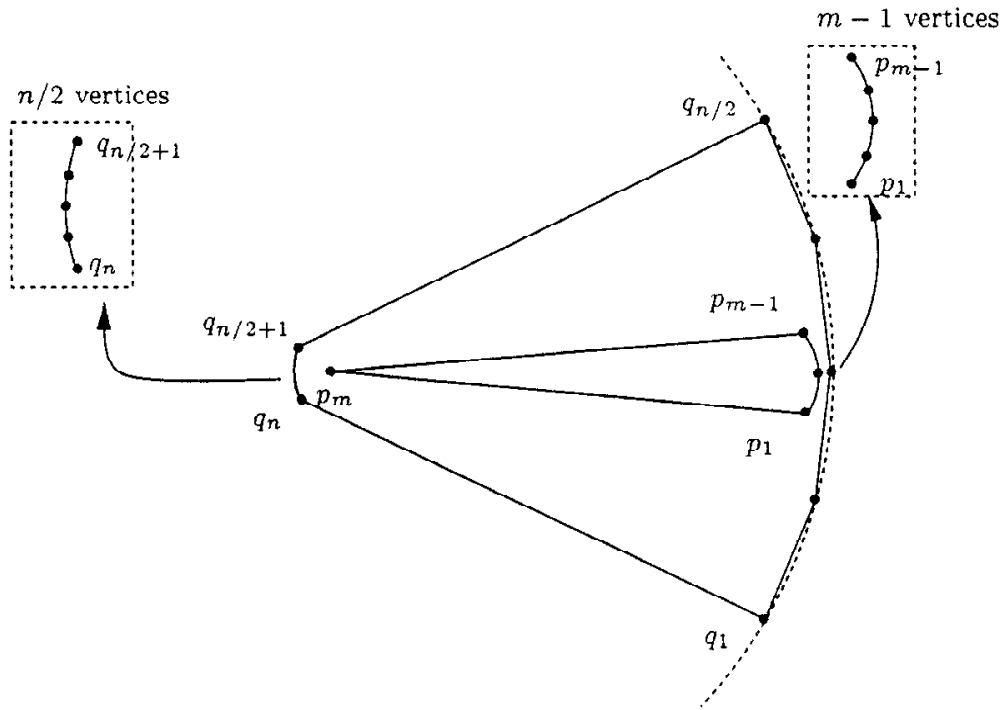

Fig. 4. Polygons $P$ and $Q$ for which there exist $\Omega\left(m n^{2}\right)$ similar placements of $P$ in $Q$ with four vertex-edge incidences per placement.

Claim. If $\varepsilon$ is chosen sufficiently small, then the following holds. For every triple $n / 2+1 \leq i \leq n, 1 \leq j<n / 2$, and $1 \leq k \leq m-2$, there is a placement of $P$ inside $Q$, using translation, rotation, and scaling, such that the vertex $p_{m}$ of $P$ coincides with the vertex $q_{i}$ of $Q$, and such that the edge $p_{k} p_{k+1}$ of $P$ coincides with the edge $q_{j} q_{j+1}$ of $Q$.

Notice that every such placement of $P$ induces four vertex-edge incidences between $P$ and $Q$, and is thus a vertex of $\mathcal{C}$.

Proof. We consider the scaling, rotation, and translation of $P$ that places $p_{k} p_{k+1}$ on the line $\ell$ supporting $q_{j} q_{j+1}$ and also places $p_{m}$ at $q_{i}$.

As in Fig. 5, let $q$ be the midpoint of edge $q_{j} q_{j+1} ; q$ is also the orthogonal projection of the origin $o$ onto the line $\ell$ supporting $q_{j} q_{j+1}$. Let $q^{\prime}$ be the projection of $p_{m}$, which is placed at $q_{i}$, onto $\ell$. Let $q^{\prime \prime}$ be the projection onto $\ell$ of $o^{\prime}$, the center of the small circle whose boundary contains the points $p_{1}, \ldots, p_{m-1}$, which is appropriately shifted with $P$. Let $q^{\prime \prime \prime}$ be the intersection of $\ell$ with the line passing through $p_{m}=q_{i}$ and $o^{\prime}$. Finally, let $s$ be the intersection of $\ell$ with the line supporting $p_{m} p_{m-1}$ (at this placement of $P$ ).

The distance from $q$ to $q^{\prime}$ is at most $\varepsilon$. The angle $q^{\prime \prime \prime} p_{m} q^{\prime}$ is the same as the angle $q^{\prime \prime \prime} o^{\prime} q^{\prime \prime}$, which, by the construction of $P$, is at most $\pi /(40 l)$. The angle $s p_{m} q^{\prime \prime \prime}$ is exactly $\pi /(40 l)$. Since the distance from $p_{m}$ to $q^{\prime}$ is at most $1+\varepsilon$, the distance from $q$ to $s$ is

$$
d(q, s) \leq d\left(q, q^{\prime}\right)+d\left(q^{\prime}, s\right) \leq \varepsilon+(1+\varepsilon) \tan \frac{\pi}{20 l} .
$$

We can choose $\varepsilon$ small enough so that

$$
\varepsilon+(1+\varepsilon) \tan \frac{\pi}{20 l}<\sin \frac{\pi}{6 l}=d\left(q, q_{j+1}\right),
$$




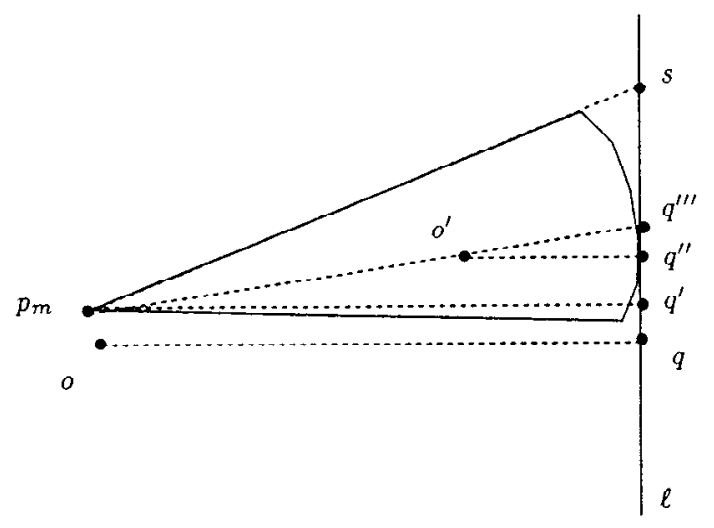

Fig. 5. Proof of claim.

which then implies that this placement of $P$ fully lies below the segment $p_{m} q_{j+1}$. An analogous argument shows that $P$ lies above the segment $p_{m} q_{j}$, so $P$ lies inside $Q$, as claimed.

We therefore obtain the following result.

Theorem 4. There exist a convex $m$-gon $P$ and another convex $n$-gon $Q$ such that there are $\Omega\left(m n^{2}\right)$ placements of similar copies of $P$ inside $Q$, each of which induces four vertex-edge incidences between $P$ and $Q$.

Remarks. (1) A weakness of the above lower-bound construction is that it only yields placements of $P$ with "degenerate" vertex-edge contacts, including a vertex-vertex contact and an edge-edge containment. Is there another construction, in which there are $\Omega\left(m n^{2}\right)$ similar placements of $P$ inside $Q$, such that at each of them four distinct vertices of $P$ touch four distinct edges of $Q$ ? This extends a similar open problem, asking for $\Omega\left(m n^{2}\right)$ congruent placements of $P$ inside $Q$, each with three contacts of distinct vertices of $P$ with distinct edges of $Q$; see [13] for details.

(2) Another open problem is whether the algorithm for finding the largest similar placement of $P$ inside $Q$ can be improved. Such an improvement could be by at most a logarithmic factor if we have to compute the entire space $\mathcal{C}$, as is implied by the above lower bound. Can we do better if we only need to compute the largest placement of $P$ ?

\section{Acknowledgments}

We are grateful to Boris Aronov and Emo Welzl for helpful discussions, and to David Jacobs and Ronen Basri for bringing Baird's representation to our attention. 


\section{References}

1. P. K. Agarwal, B. Aronov, and M. Sharir, Motion planning for a convex polygon in a polygonal environment, unpublished manuscript.

2. F. Avnaim and J.-D. Boissonnat, Simultaneous containment of several polygons, Proc. 3rd Annu. ACM Symp. Comput. Geom., 1987, pp. 242-250.

3. F. Avnaim and J.-D. Boissonnat, Polygon placement under translation and rotation, Proc. 5th Symp. Theoret. Aspects Comput. Sci., Lecture Notes in Computer Science, vol. 294, Springer-Verlag, Berlin, 1988, pp. 322-333.

4. H. S. Baird, Model-Based Image Matching Using Location, Distinguished Dissertation Series, MIT Press, Cambridge, MA, 1984.

5. R. Basri and D. Jacobs, Recognition using region correspondences, Proc. 5th Internat. Conf. Comput. Vision, 1995, pp. 8-13.

6. B. Chazelle, The polygon containment problem, in Advances in Computing Research, vol. 1 (F. P. Preparata, ed.), JAI Press, London, 1983, pp. 1-33.

7. B. Chazelle and J. Friedman, A deterministic view of random sampling and its use in geometry, Combinatorica 10 (1990), 229-249.

8. L. P. Chew and K. Kedem, A convex polygon among polygonal obstacles: Placement and high-clearance motion, Comput. Geom. Theory Appl. 3 (1993), 59-89.

9. K. M. Daniels and V. J. Milenkovic, Multiple translational containment: Approximate and exact algorithms, Proc. 6th ACM-SIAM Symp. on Discrete Algorithms, 1995, pp. 205-214.

10. L. Guibas, L. Ramshaw, and J. Stolfi, A kinetic framework for computational geometry, Proc. 24th Annu. IEEE Symp. Found. Comput. Sci., 1983, pp. 100-111.

11. J. Matoušek, M. Sharir, and E. Welzl, A subexponential bound for linear programming and related problems, Algorithmica 16 (1996), 498-516.

12. V. J. Milenkovic, Translational polygon containment and minimal enclosure using linear programming based restriction, Proc. 28th ACM Symp. Theory Comput., 1996, pp. 109-118.

13. D. Parson and C. Torras, The combinatorics of overlapping convex polygons in contact, Proc. 4th Canad. Conf. Comput. Geom., 1992, pp. 83-92.

14. F. Preparata and S. Hong, Convex hulls of finite sets of points in two and three dimensions, Comm. ACM 20 (1977), 87-93.

15. F. P. Preparata and M. I. Shamos, Computational Geometry: An Introduction, Springer-Verlag, New York, 1985.

16. M. Sharir and S. Toledo, Extremal polygon containment problems, Comput. Geom. Theory Appl. 4 (1994), 99-118.

Received December 11, 1995, and in revised form March 3, 1997. 